\title{
Effectiveness of the interventions in preventing the progression of pre-frailty and frailty in older adults: a systematic review protocol
}

\author{
João Apóstolo ${ }^{1}$ \\ Richard Cooke ${ }^{2}$ \\ Elzbieta Bobrowicz-Campos ${ }^{1}$ \\ Silvina Santana ${ }^{3}$ \\ Maura Marcucci ${ }^{4}$ \\ Antonio Cano 5 \\ Miriam Vollenbroek 6 \\ Carol Holland ${ }^{2}$
}

1. Health Sciences Research Unit: Nursing, Nursing School of Coimbra, Portugal Centre for Evidence Based Practice: a Collaborating Centre of the Joanna Briggs Institute

2. Aston Research Centre for Healthy Ageing (ARCHA), Aston University, Birmingham, United Kingdom

3. Economics, Management and Industrial Engineering Department, University of Aveiro, Portugal

4. Geriatric Unit, Fondazione IRCCS Ca' Granda Ospedale Maggiore Policlinico and Department of Clinical Science and Community Health, University of Milan, Milan, Italy

5. Department Of Paediatrics, Obstetrics and Gynaecology, Universitat de Valência, Spain

6. Roessingh Research and Development, The Netherlands

Corresponding author:

João Apóstolo apostolo@esenfc.pt

\section{Review question/objective}

The objective of this review is to identify the effectiveness of the interventions in preventing progression of pre-frailty and frailty in older adults.

More specifically, the review questions are:

- What is the effectiveness of interventions in preventing or reducing frailty in older adults?

- How does effectiveness vary with degree of frailty?

- Are there factors that influence the effectiveness of interventions?

- What is the economic feasibility of interventions for pre-frailty and frailty? 


\section{Background}

Frailty is an age-related state of high vulnerability to adverse health outcomes after a stressor event ${ }^{1-6}$, predisposing the individuals to falls and fractures, disability, dependency, hospitalization and institutional placement, and ultimately death. ${ }^{1}$ It can be preceded by, but also occurs in the absence of, chronic disease. ${ }^{3,5,7}$ According to some authors, this clinical condition results from decrease in reserves across multiple physiological systems that are normally responsible for healthy adaptation to stress. ${ }^{1,8,9}$ Alternatively, it is considered that frailty is due to the critical accumulation of dysregulation in important signaling pathways and subsequent depletion of homeostatic reserve and resiliency. ${ }^{1,10,11}$ Other authors describe this state of increased vulnerability as associated with a reduced capacity to compensate aging-related molecular and cellular damage. ${ }^{2}$ Independently of pathophysiological conceptualization, it is assumed that frailty is a dynamic process that leads to a spiraling decline in various functional domains that exacerbates the risk of geriatric syndromes. ${ }^{1,3,5,6}$

The phenotypic markers of frailty include global weakness with low muscle strength (e.g. poor grip strength), overall slowness (particularly in gait), decreased balance and mobility, fatigability or exhaustion, low physical activity and involuntary weight loss. ${ }^{1,3,5,8}$ For diagnostic purposes, at least three of these components must be observed. ${ }^{8}$ The presence of only one or two of them is considered as an indicator of the state of pre-frailty. From a broader perspective, it is assumed that frailty can also manifest through cognitive impairment, ${ }^{12-16}$ although, according to evidence, the decline in cognition is very selective, being limited to executive functions, attention, verbal fluency and processing of speed. It is also well documented that frail elderlies manifest some impairment in activities of daily living and report significant reduction in quality of life. ${ }^{12,17}$ Furthermore, recent studies have shown that frailty may be related to mood change, ${ }^{16,18}$ although the nature of this association, as well as its relevance to the frailty construct, needs to be clarified. ${ }^{17}$

Regarding the prevalence of frailty, systematic comparison of numerous studies ${ }^{19}$ shows that frailty in community-dwelling adults aged 65 and over varies from $4 \%$ to $17 \%$. In case of pre-frailty, the frequency varies between $19 \%$ and $53 \%$ in different studies. ${ }^{19}$ The differences in estimates due to differences in the operational definition of frailty (based on physical markers or incorporating a broader multidimensional approach) and study populations (e.g. the results of epidemiological studies can be affected by demographic variables, such as age and gender, as well as the presence of chronic disease or other comorbid conditions).

Because of the frequency of its occurrence and the severity of its consequences, frailty is seen as a threatening condition for older adults, requiring attention from health care professionals, social care practitioners, researchers and policy-makers. ${ }^{3,6,19}$ The implications of the involvement of these agents can be observed at various levels, with issues related to improving prognosis and preventing the progression from pre-frail to frail being of the greatest interest and relevance. In relations to interventions, attempts to manage adverse consequences of frailty are often focused on minimization of risks of disability and dependency, or on the treatment of underlying conditions and symptoms. For complementary approaches, frailty management involves the development of coping strategies necessary to control potential stress factors or to minimize their impact. ${ }^{3,20}$ So far, various types of intervention have been proposed, among which are physical activity, psychosocial intervention, health and social care provision, cognitive stimulation, nutrition, medication/medical maintenance and adherence focused interventions, and multifactorial intervention. The results of studies conducted in 
this area have indicated that treating frailty in older adults is a realistic therapeutic goal. ${ }^{21-24}$ However, it is still hard to determine how effective these types of intervention are and how efficiency can be influenced by other factors, for example, severity of the clinical condition. Another issue requiring clarification is the effectiveness of interventions in terms of drug prescription and changes in analytical parameters..$^{25,26}$ The focus of attention should also be directed to economic data, namely, costs relative to benefits and/or savings associated with implementing the interventions for pre-frailty and frailty. ${ }^{27}$

A preliminary search of the JBI Database of Systematic Reviews and Implementation Reports, the Cochrane Database of Systematic Reviews, Prospero, CINAHL, and Medline has revealed that there are currently no systematic review (neither published nor in progress) on clinical/medical and economic effectiveness of interventions to prevent or reduce frailty in older adults.

Therefore, it is necessary for a systematic review to be conducted to examine the effectiveness of the interventions to prevent the progression of pre-frailty and frailty in older adults, involving a critical analysis based on scientific evidence.

\section{Keywords}

frail elderly; pre-frailty; frailty

\section{Inclusion criteria}

\section{Types of participants}

This review will consider studies that include older adults (female and male) aged 65 years and over, explicitly identified as pre-frail or frail by the researchers or associated medical professionals according to a pre-specified scale or index, and who have received health care and support services in any type of setting (primary care, nursing homes, hospitals).

This review will exclude studies that:

- Include participants who have been selected because they have one specific illness

- Consider people with a terminal diagnosis only.

\section{Types of intervention(s)/phenomena of interest}

The clinical/medical component of the review will consider studies that evaluate any type of interventions to prevent the progression of pre-frailty and frailty in older adults. These interventions will include, but will not be limited to, physical activity, multifactorial intervention, psychosocial intervention, health and social care provision, and cognitive, nutrition or medication/medical maintenance and adherence focused interventions.

The economic component of the review will consider studies that have performed any type of health economic analysis of interventions to prevent the progression of pre-frailty and frailty in older adults.

The comparator for both clinical/medical and economic components will be implementing usual care, alternative therapeutic interventions or no intervention.

\section{Types of outcomes}

Primary outcomes:

- Frailty as indicated by any validated scale or measurement or index. 
Secondary outcomes:

i. Cognition, quality of life, quality-adjusted life year (QALY), activities of daily living (ADL), instrumental activities of daily living (IADL), caregiver burden, functional capacity, depression, self-perceived health and social engagement, as assessed by any validated scale or measurement or index.

ii. Drugs and prescriptions, analytical parameters, falls and fractures, mortality, hospitalization, institutionalization and comorbidities.

iii. Costs and/or costs relative to benefits and/or savings associated with implementing the interventions for pre-frailty and frailty.

\section{Types of studies}

The clinical / medical component of the review will consider randomized controlled trials (RCTs), non-randomized trials and quasi-experimental studies related to the effectiveness of interventions for pre-frailty and frailty for inclusion. In the absence of RCTs, non-randomized trials, quasi-experimental studies and other research designs of a quantitative nature, such as cohort studies, will be considered for inclusion.

The economic component of the review will consider cost effectiveness, cost benefit, cost minimization or cost utility studies. Any quantitative study measuring clinical effectiveness that incorporates economic data will be considered. Studies where the effectiveness of the intervention on frailty measures is not measured will be excluded.

\section{Search strategy}

The search strategy aims to find both published and unpublished studies. A three-step search strategy will be utilized in this review. An initial limited search of MEDLINE via EBSCOhost Web and CINAHL will be undertaken followed by an analysis of text words contained in the title and abstract, and of the index terms used to describe the article. A second search using all identified keywords and index terms will then be undertaken across all included databases. Thirdly, the reference lists of all identified reports and articles will be searched for additional studies. Studies published in English, Portuguese, Spanish, Italian and Dutch will be considered for inclusion in this review. Studies published from 2001 will be considered for inclusion in this review. This timeline was selected because 2001 is the year of publication of Fried's paper ${ }^{8}$ that showed to be seminal for research on frailty.

The databases to be searched include:

CINAHL

MEDLINE

SCOPUS

EMBASE

Cochrane Central Register of Controlled Trials

Scielo.

The search for unpublished studies will include: 


\section{ProQuest Theses and Dissertations}

OpenGrey

Banco de teses da CAPES (www.capes.gov.br)

Dissertation Abstracts Online (e-Thos).

Initial keywords to be used will be frailty, elder ${ }^{*}$, old ${ }^{*}$, intervention*

During the process of conducting the search, various terminologies and spellings of the keywords will be taken into consideration as they might affect the identification of relevant studies.

\section{Assessment of methodological quality}

Quantitative papers selected for retrieval will be assessed by two independent reviewers for methodological validity prior to inclusion in the review using standardized critical appraisal instruments from the Joanna Briggs Institute Meta-Analysis of Statistics Assessment and Review Instrument (JBI-MAStARI) (Appendix I).

Economic papers selected for retrieval will be assessed by two independent reviewers for methodological validity prior to inclusion in the review using standardized critical appraisal instruments from the Joanna Briggs Institute Analysis of Cost, Technology and Utilization Assessment and Review Instrument (JBI-ACTUARI) (Appendix I).

Any disagreements that arise between the reviewers will be resolved through discussion, or with a third reviewer.

\section{Data extraction}

Quantitative data will be extracted from papers included in the review independently by two reviewers using the standardized data extraction tool from JBI-MAStARI (Appendix II). The data extracted will include specific details about the interventions, populations, study methods and outcomes of significance to the review question and specific objectives.

Economic data will be extracted from papers included in the review using the standardized data extraction tool from JBI-ACTUARI (Appendix II). The data extracted will include specific details about the interventions, populations, study methods and outcomes of significance to the review question and specific objectives.

If there is missing information or data that needs clarification, the authors of primary studies will be contacted. Any disagreements that arise between the reviewers will be resolved through discussion or with a third reviewer.

\section{Data synthesis}

Quantitative papers will, where possible, be pooled in statistical meta-analysis using JBI-MAStARI. All results will be subject to double data entry. Effect sizes expressed as odds ratio (for categorical data) and weighted mean differences (for continuous data) and their $95 \%$ confidence intervals will be calculated for analysis. Heterogeneity will be assessed statistically using the standard Chi-square. Plausible explanation for variations in treatment effects will be explored using subgroup analyses, whenever possible, based on frailty levels and settings included in the studies. Where statistical 
pooling is not possible the findings will be presented in narrative form including tables and figures to aid in data presentation where appropriate.

Economic findings will, where possible, be pooled using JBI-ACTUARI and presented in a tabular summary. Where this is not possible, findings will be presented in narrative form.

\section{Conflicts of interest}

The authors declare that there are no conflicts of interest.

\section{Acknowledgements}

This protocol is part of the project / joint action '664367 / FOCUS' which has received funding from the European Union's Health Programme (2014-2020).

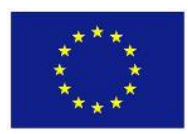

\section{$\therefore$ FOCUS}

The authors acknowledge the contribution of the following Focus Group members: Alessandro Nobili and Barbara D'Avanzo (IRCCS Istituto Di RicercheFarmacologiche "Mario Negri"), Ana González Segura and Enrique de la Cruz Martínez (EVERIS Spain S.L.U), Ana M. Martínez-Arroyo, Vicente Gil and Vicente Llorens (ESAM Tecnología S.L.), Donata Kurpas and Maria Bujnowska (Wroclaw Medical University), Federico Germini (Fondazione IRCCS Cà Granda - Ospedale Maggiore Policlinico), James Brown and Rachel Shaw (Aston Research Centre for Healthy Ageing, Aston University) and Lex van Velsen (Roessingh Research and Development) who were involved in decision-making and planning of the FOCUS project, and development of PICO questions and structuring of PICO components for this protocol.

The authors also thank Daniela Cardoso for her contribution to the protocol development. 


\section{References}

1. Fried LP, Ferrucci L, Darer J, et al. Untangling the concepts of disability, frailty, and comorbidity: implications for improved targeting and care. J Gerontol A Biol Sci Med Sci 2004;59(3):255-263.

2. Clegg A, Young J, lliffe S, et al. Frailty in elderly people. Lancet 2013; 381(9868):752-762.

3. Topinková E. Aging, Disability and Frailty. Ann Nutr Metab 2008;52(suppl 1):6-11.

4. Lang PO, Michel JP, Zekry D. Frailty Syndrome: A Transitional State in a Dynamic Process. Gerontology 2009;55(5):539-549.

5. Bergman H, Ferrucci L, Guralnik J, et al. Frailty: an emerging research and clinical paradigm - issues and controversies. J. Gerontol. A: Biol. Sci. Med. Sci. 2007;62(7):731-737.

6. Lipsitz LA: Dynamic models for the study of frailty. Mech Ageing Dev 2008;129(11):675-676.

7. Rockwood K, Mitnitski A. Frailty in relation to the accumulation of deficits. J Gerontol A Biol Sci Med Sci 2007;62(7):722-727.

8. Fried LP, Tangen CM, Walston J, et al. Frailty in older adults: evidence for a phenotype. J GerontolBiol Med Sci 2001;56(3):M146-M156.

9. Lang PO, Michel JP, Zekry D. Frailty Syndrome: A transitional State in a Dynamic Process. Gerontology 2009;55(5):539-549.

10. Ferrucci L, Windham BG, Fried LP. Frailty in older persons. Genus 2005;61(1):39-53.

11. Varadhan R, Seplaki CS, Xue QL, et al. Stimulus-response paradigm for characterizing the loss of resilience in homeostatic regulation associated with frailty. Mech Ageing Dev 2008;129(11):666-670.

12. Langlois F, Vu TTM, Kergoat MJ, et al. The multiple dimensions of frailty: physical capacity, cognition, and quality of life. International Psychogeriatrics 2012;24(9):1429-1436.

13. Lin F, Roiland R, Chen DG, et al. Linking cognition and frailty in middle and old age: metabolic syndrome matters. Int J Geriatr Psychiatry. 2015;30(1):64-71.

14. Halil M, Kizilarslanoglu MC, Kuyumcu ME, et al. Cognitive aspects of frailty: mechanisms behind the link between frailty and cognitive impairment. J Nutr Health Aging 2015;19(3):276-283.

15. Robertson DA, Savva GM, Kenny RA. Frailty and cognitive impairment - A review of the evidence and causal mechanisms. Ageing Research Reviews 2013;12(4):840-851.

16. Ávila-Funes, JA, Amieva $H$, Barberger-Gateau $P$, et al. Cognitive Impairment Improves the Predictive Validity of the Phenotype of Frailty for Adverse Health Outcomes: The Three-City Study. J Am Geriatr Soc 2009;57(3):453-461.

17. Sternberg SA, Schwartz AW, Karunananthan S, et al. The identification of frailty: A systematic literature review. Progress in Geriatrics 2011;59(11):2129-2139.

18. Collard, RM, Comijs HC, Naarding P, et al. Frailty as a Predictor of the Incidence and Course of Depressed Mood. J Am Med Direct Ass 2015;16(6):509-514. 
19. Collard RM, Boter $\mathrm{H}$, Schoevers RA, et al. Prevalence of frailty in community-dwelling older persons: A systematic review. J Am Geriatr Soc 2012;60(8):1487-1492.

20. Andreasen PT, Lund $\mathrm{H}$, Aadahl M, et al. The experience of daily life of acutely admitted frail elderly patients one week after discharge from the hospital. Int J Qualitative Stud Health Well-being 2015;10:27370.

21. Cameron ID, Fairhall N, Langron C, et al. A multifactorial interdisciplinary intervention reduces frailty in older people: randomized trial. BC Med 2013;11:65.

22. Cesari M, Vellas B, Hsu F-C, et al. A Physical Activity Intervention to Treat the Frailty Syndrome in Older Persons - Results From the LIFE-P Study. J Gerontol A Biol Sci Med Sci 2015;70(2):216-222.

23. Pulignano G, Del Sindaco D, Di Lenarda A, et al. Usefulness of frailty profile for targeting older heart failure patients in disease management programs: a cost-effectiveness, pilot study. J Cardiovasc Med 2010;11(10):739-747.

24. Eklund K, Wilhelmson K, Gustafsson $\mathrm{H}$, et al. One-year outcome of frailty indicators and activities of daily living following the randomized controlled trial; "Continuum of care for frail older people". BMC Geriatrics 2013;13:76

25. Bastos-Barbosa RG, Ferriolli E, Coelho EB, et al. Association of frailty syndrome in the elderly with higher blood pressure and other cardiovascular risk factors. Am $J$ Hypertens. 2012;25(11):1156-1161.

26. Peklar J, O'Halloran AM, Maidment ID, et al. Sedative Load and Frailty Among Community-Dwelling Population Aged $\geq 65$ Years. J Am Med Dir Assoc 2015;16(4):282-289.

27. Fairhall N, Sherrington C, Kurrle S, et al. Economic Evaluation of a Multifactorial, Interdisciplinary Intervention Versus Usual Care to Reduce Frailty in Frail Older People. J Am Med Dir Assoc 2015;16(1):41-48. 


\section{Appendix I: Appraisal instruments \\ MAStARI appraisal instrument}

\section{JBI Critical Appraisal Checklist for Randomised Control / Pseudo-randomised Trial}



Overall appraisal: $\quad$ Include $\square \quad$ Exclude $\square \quad$ Seek further info.

Comments (Including reason for exclusion) 


\section{JBI Critical Appraisal Checklist for Descriptive / Case Series}

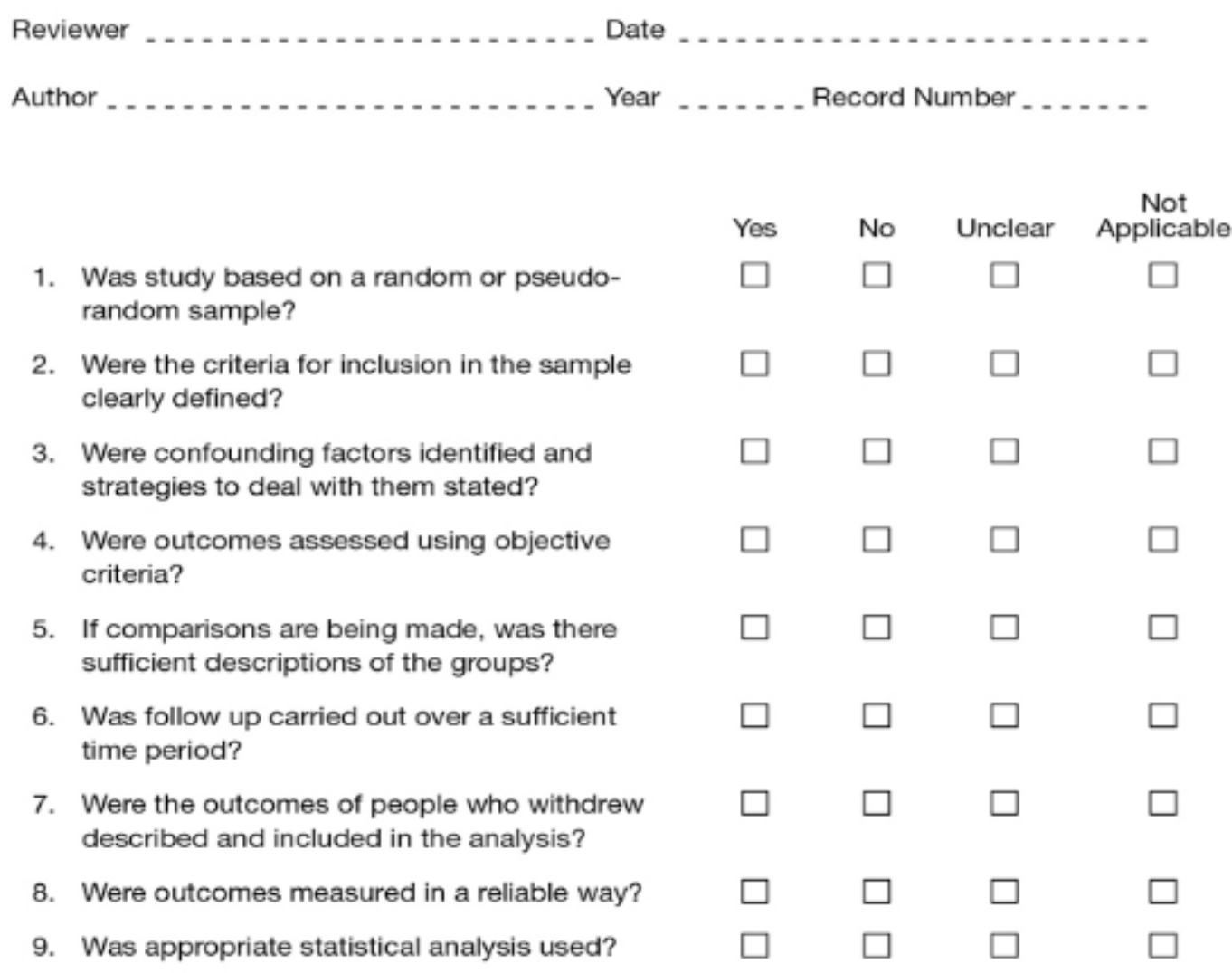

Overall appraisal: $\quad$ Include $\square \quad$ Exclude $\square \quad$ Seek further info

Comments (Including reason for exclusion) 


\section{JBI Critical Appraisal Checklist for Comparable Cohort/ Case Control}

\begin{tabular}{|c|c|c|c|c|}
\hline \multicolumn{5}{|c|}{ Author } \\
\hline & Yes & No & Unclear & Not Applicable \\
\hline $\begin{array}{l}\text { 1. Is sample representative of patients } \\
\text { in the population as a whole? }\end{array}$ & $\square$ & $\square$ & $\square$ & $\square$ \\
\hline $\begin{array}{l}\text { 2. Are the patients at a similar point in } \\
\text { the course of their condition/illness? }\end{array}$ & $\square$ & $\square$ & $\square$ & $\square$ \\
\hline $\begin{array}{l}\text { 3. Has bias been minimised in relation } \\
\text { to selection of cases and of } \\
\text { controls? }\end{array}$ & $\square$ & $\square$ & $\square$ & $\square$ \\
\hline $\begin{array}{l}\text { 4. Are confounding factors identified } \\
\text { and strategies to deal with them } \\
\text { stated? }\end{array}$ & $\square$ & $\square$ & $\square$ & $\square$ \\
\hline $\begin{array}{l}\text { 5. Are outcomes assessed using } \\
\text { objective criteria? }\end{array}$ & $\square$ & $\square$ & $\square$ & $\square$ \\
\hline $\begin{array}{l}\text { 6. Was follow up carried out over a } \\
\text { sufficient time period? }\end{array}$ & $\square$ & $\square$ & $\square$ & $\square$ \\
\hline $\begin{array}{l}\text { 7. Were the outcomes of people who } \\
\text { withdrew described and included in } \\
\text { the analysis? }\end{array}$ & $\square$ & $\square$ & $\square$ & $\square$ \\
\hline $\begin{array}{l}\text { 8. Were outcomes measured in a } \\
\text { reliable way? }\end{array}$ & $\square$ & $\square$ & $\square$ & $\square$ \\
\hline $\begin{array}{l}\text { 9. Was appropriate statistical analysis } \\
\text { used? }\end{array}$ & $\square$ & $\square$ & $\square$ & $\square$ \\
\hline Overall appraisal: & Exclude & $\square$ & $\mathrm{Se}$ & further info. $\square$ \\
\hline
\end{tabular}




\section{ACTUARI appraisal instrument}

\section{JBI Critical Appraisal Checklist for Economic Evaluations}

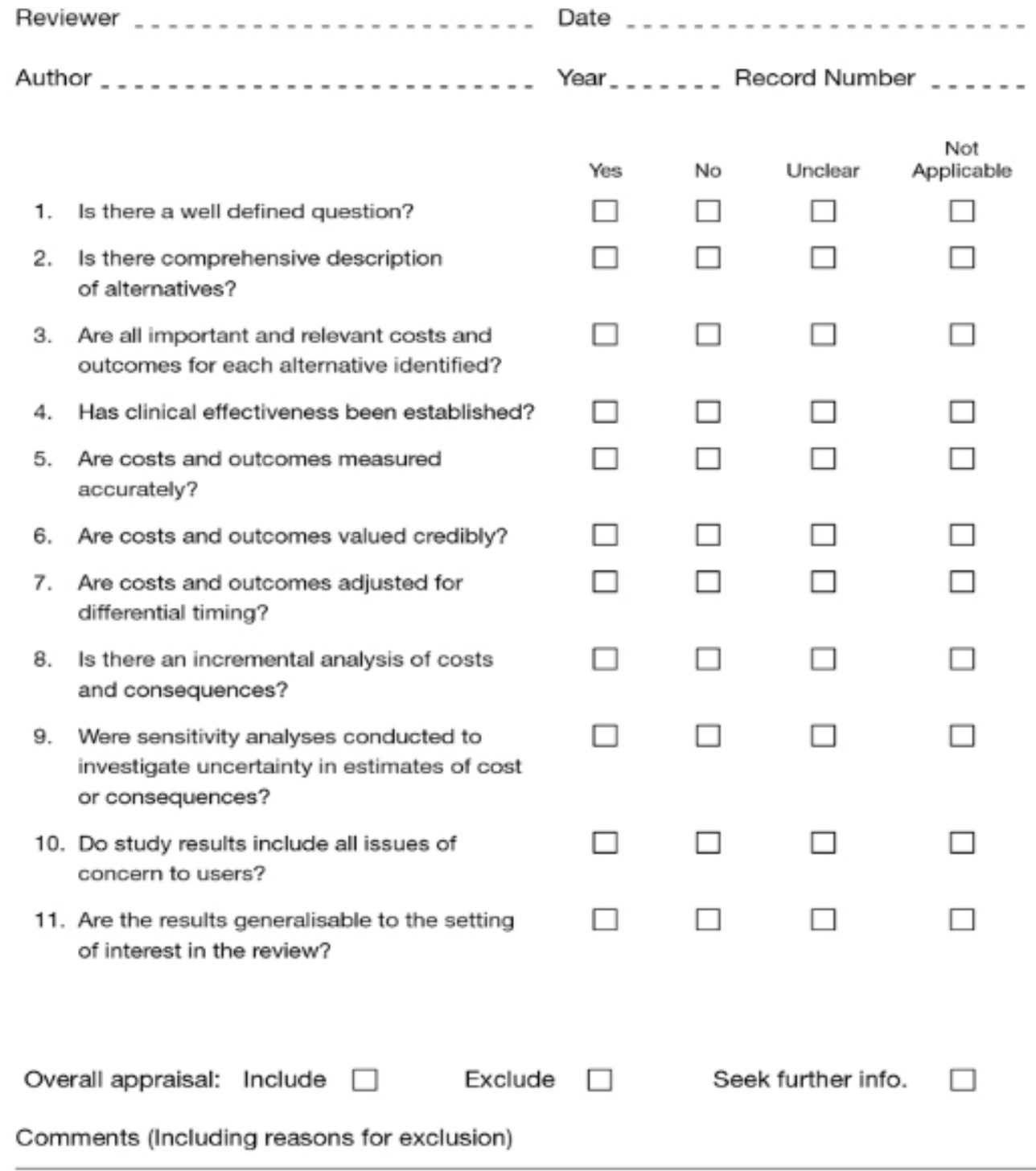




\section{Appendix II: Data extraction instruments}

MAStARI data extraction instrument

\section{JBI Data Extraction Form for Experimental / Observational Studies}

\begin{tabular}{|c|c|c|c|c|c|}
\hline \multicolumn{6}{|c|}{ Reviewer _. } \\
\hline \multicolumn{6}{|c|}{ 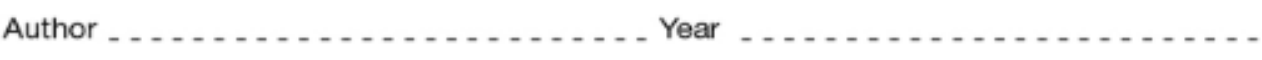 } \\
\hline \multicolumn{6}{|l|}{ Journal_. } \\
\hline \multicolumn{6}{|l|}{ Study Method } \\
\hline RCT & $\square$ & Quasi-RCT & $\square$ & Longitudinal & $\square$ \\
\hline Retrospective & $\square$ & Observational & $\square$ & Other & $\square$ \\
\hline \multicolumn{6}{|l|}{ Participants } \\
\hline Setting & & & & & \\
\hline
\end{tabular}

\section{Sample size}

Group A Group B

\section{Interventions}

Intervention A

Intervention B

Authors Conclusions:

Reviewers Conclusions: 


\section{Study results}

\section{Dichotomous data}

\begin{tabular}{|l|l|l|}
\hline Outcome & $\begin{array}{c}\text { Intervention ( } \\
\text { number/total number }\end{array}$ & $\begin{array}{c}\text { Intervention ( ) } \\
\text { number/total number }\end{array}$ \\
\hline & & \\
\hline & & \\
\hline & & \\
\hline & & \\
\hline & & \\
\hline
\end{tabular}

Continuous data

\begin{tabular}{|l|l|l|}
\hline Outcome & $\begin{array}{c}\text { Intervention ( ) } \\
\text { number/total number }\end{array}$ & $\begin{array}{c}\text { Intervention ( ) } \\
\text { number/total number }\end{array}$ \\
\hline & & \\
\hline & & \\
\hline & & \\
\hline & & \\
\hline & & \\
\hline
\end{tabular}




\section{ACTUARI data extraction instrument}

\section{JBI Data Extraction Form for Economic Evaluations}

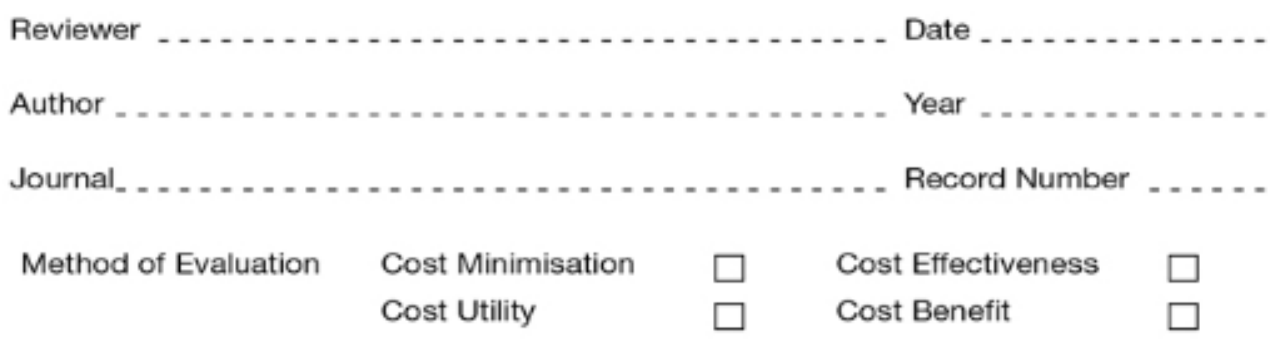

Interventions

Comparator

Setting

Geographical

Participants

Source of effectiveness data

Authors Conclusions

Reviewers Comments 


\section{Clinical Effectiveness Results}

Study design

Year range of primary studies

Analysis used

Clinical outcome results

\section{Economic Effectiveness results}

Date/s of economic data

Modeling used

Measure of benefits used in economic evaluation

Direct costs

Indirect costs

Currency

Statistical analysis

Estimated benefits used in EE

Cost results

Synthesis of costs and results

\section{Outcome category}

\begin{tabular}{|c|c|c|c|c|c|c|c|}
\hline & \multicolumn{6}{|c|}{ Clinical effectiveness } \\
\hline & & & 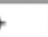 & & 5 & & \\
\hline \multirow{3}{*}{ Cost } & + & 0 & A & 0 & B & 0 & C \\
\hline & 0 & 0 & D & 0 & $E$ & 0 & $\mathrm{~F}$ \\
\hline & - & 0 & $G$ & 0 & $\mathrm{H}$ & 0 & 1 \\
\hline
\end{tabular}

\begin{tabular}{|lll|}
\hline & \multicolumn{2}{c|}{ Key } \\
& Effectiveness & Cost \\
+ & Better & Lower \\
0 & Equal & Equal \\
- & Poorer & Higher \\
\hline
\end{tabular}

